\title{
Correlation Analysis of Four Blood Coagulation Items with Blood Lipids and Blood Glucose in Stroke Patients with Type 2 Diabetes
}

\author{
Yue Wang, Xue Liang \\ Institute of Integrated Chinese and Western Medicine, Basic Medical College of Qingdao University, Qingdao, China \\ Email: 121170769@qq.com
}

How to cite this paper: Wang, Y. and Liang, X. (2020) Correlation Analysis of Four Blood Coagulation Items with Blood Lipids and Blood Glucose in Stroke Patients with Type 2 Diabetes. Open Access Library Journal, 7: e7042.

https://doi.org/10.4236/oalib.1107042

Received: November 26, 2020

Accepted: December 28, 2020

Published: December 31, 2020

Copyright $\odot 2020$ by author(s) and Open Access Library Inc.

This work is licensed under the Creative Commons Attribution International License (CC BY 4.0).

http://creativecommons.org/licenses/by/4.0/

\begin{abstract}
Objective: To investigate the relationship between the four blood coagulation items and blood glucose and blood lipids in stroke patients with type 2 diabetes. Methods: According to whether they had type 2 diabetes, 134 stroke patients were divided into the observation group $(n=62)$ with stroke and type 2 diabetes and the control group without type 2 diabetes $(n=72)$, and the blood coagulation of each group was compared. Results: There was no significant difference in the four coagulation items between the two groups of patients, and hyperglycemia did not seem to have a significant effect on this. Conclusion: The coagulation changes in stroke patients may be greatly affected by the acute onset of stroke, and the impact of type 2 diabetes may be chronic and insignificant.
\end{abstract}

\section{Subject Areas}

Neurology

\section{Keywords}

Stroke, Type 2 Diabetes, Coagulation

\section{Introduction}

Ischemic stroke is a disease that forms thrombosis and causes embolism. The imbalance of the blood coagulation system and the fibrinolytic system is one of the main causes of thrombosis. The four indicators of coagulation as important indicators for judging the changes in the body's hemostasis system have received extensive clinical attention. Previous studies have shown that hyperglycemia is related to coagulation disorders [1], and patients with type 2 diabetes have vas- 
cular endothelial damage, which causes platelet activation and the hypercoagulable state of the blood [2]. Therefore, in order to prevent further complications after stroke and type 2 diabetes, improve the quality of life of patients, and understand the blood coagulation status of stroke patients with type 2 diabetes, this study measures stroke. The levels of blood glucose, blood lipids and blood coagulation in patients with type 2 diabetes were observed to observe the changes of the 4 items of blood coagulation, and the clinical significance of the changes of the 4 items of blood coagulation in patients with increased blood sugar levels.

\section{Materials and Methods}

\subsection{Clinical Data}

Collect 134 stroke patients diagnosed and treated in the Affiliated Hospital of Qingdao University from 2018 to 2019. According to whether stroke patients have type 2 diabetes, they are divided into an observation group with type 2 diabetes $(n=62)$ and a control group without type 2 diabetes $(n=72)$, there was no difference in age between the two groups $(65.88 \pm 10.17$ vs. $64.97 \pm 8.61)$.

\subsection{Method}

Inclusion criteria: 1) Patients meeting the diagnostic criteria for stroke; 2) $\mathrm{Pa}$ tients meeting the diagnostic criteria for type 2 diabetes

Exclusion criteria: 1) stroke patients with other diseases.

Laboratory testing: The subjects of each group were admitted to the hospital in the early morning on an empty stomach to collect peripheral venous blood. The laboratory of our hospital performed laboratory tests. The automatic biochemical analyzer (Hitachi-7600, Japan) measured four blood coagulation items in the two groups: coagulation Proenzyme time (PT), thromboplastin time (APTT), fibrinogen (FIB), thrombin time (TT), blood glucose (GLU), low density lipoprotein cholesterol (LDL), high density lipoprotein cholesterol (HDL), triglycerides (TG), cholesterol (CHOL).

\subsection{Statistical Processing}

SPSS21.0 statistical software was used for data processing. The comparison of means between groups was performed by $\mathrm{t}$-test or rank-sum test. The correlation analysis was performed by preseason correlation analysis. $P<0.05$ was considered statistically significant.

\section{Result}

Comparing the four items of basic blood glucose, blood lipids and coagulation between the two groups of patients, only the blood glucose was significantly different $(t=6.17, P<0.001)$, and there were no significant differences in other indicators (See Table 1 and Table 2). 
Correlation analysis, only PT is correlated with LDL $(r=0.29, P=0.001)$ and $\operatorname{CHOL}(r=0.23, P=0.009)$ (See Table 3 and Figure 1 ).

Table 1. Comparison of blood glucose and blood lipids between the two groups (mmol/L).

\begin{tabular}{cccccc}
\hline & GLU & LDL & HDL & TG & CHOL \\
\hline Observation group & $7.27 \pm 2.67$ & $0.92 \pm 0.09$ & $1.23(0.36)$ & $3.25(3.04)$ & $1.11(0.26)$ \\
Control group & $5.14 \pm 1.13$ & $0.94 \pm 0.13$ & $1.20(0.54)$ & $3.32(1.92)$ & $1.13(0.59)$ \\
$t$ or $Z$ & 6.17 & -0.21 & 0.07 & -0.02 & -0.52 \\
$P$ & $<0.001$ & 0.83 & 0.94 & 0.99 & 0.60 \\
\hline
\end{tabular}

Table 2. Comparison of four coagulation items between the two groups.

\begin{tabular}{ccccc}
\hline & PT $(\mathrm{s})$ & APTT $(\mathrm{s})$ & FIB $(\mathrm{g} / \mathrm{L})$ & TT $(\mathrm{s})$ \\
\hline Observation group & $0.92 \pm 0.09$ & $1.23(0.36)$ & $3.25(3.04)$ & $1.11(0.26)$ \\
Control group & $0.94 \pm 0.13$ & $1.20(0.54)$ & $3.32(1.92)$ & $1.13(0.59)$ \\
$t$ or $z$ & -0.54 & -0.13 & -0.02 & -0.37 \\
$P$ & 0.59 & 0.90 & 0.99 & 0.71 \\
\hline
\end{tabular}

Table 3. Correlation analysis of four blood coagulation items with blood sugar and blood lipids.

\begin{tabular}{ccccc}
\hline & PT & APTT & FIB & TT \\
\hline GLU & -0.20 & -0.06 & 0.10 & 0.09 \\
LDL & $0.29^{*}$ & -0.08 & 0.03 & -0.04 \\
HDL & -0.18 & 0.02 & 0.12 & 0.07 \\
TG & -0.10 & 0.10 & 0.04 & -0.01 \\
CHOL & $0.23^{*}$ & -0.04 & 0.01 & -0.01 \\
\hline
\end{tabular}

${ }^{*} P<0.05$.

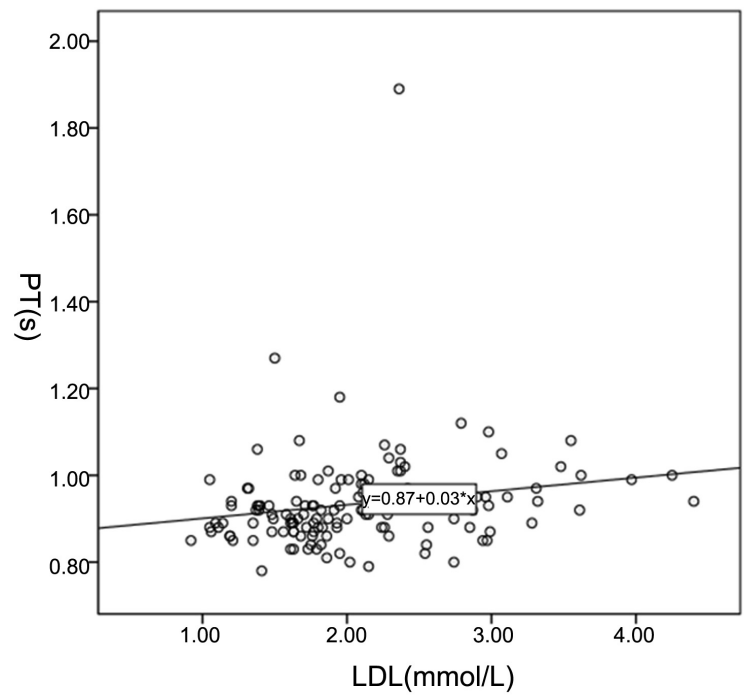

(a)

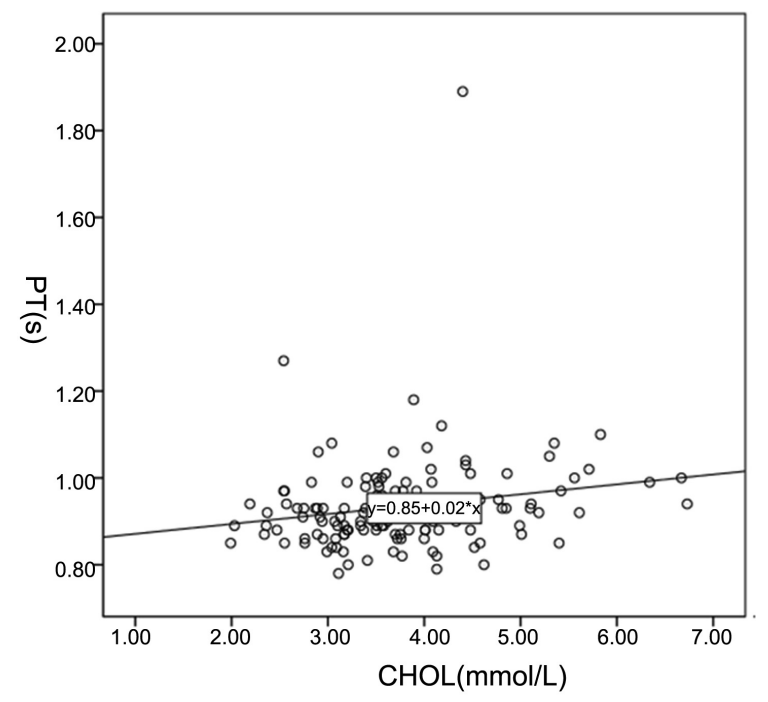

(b)

Figure 1. Correlation between PT and LDL (a) and CHOL (b). 


\section{Conclusion}

A study of elderly patients with type 2 diabetes showed that PT and APTT were shorter than those in the healthy control group, and the PT and APTT of the complication group were lower than those of the non-complication group [3]. Lin et al. [4]'s study on patients with type 2 diabetes also showed that PT and APTT of type 2 diabetes patients with or without vascular complications decreased significantly, and the coagulation indexes PT, APTT, and TT of the group with vascular disease were significantly lower than those in the group without vascular disease. And the level of FIB increased significantly. Studies have shown that lipid metabolism disorders are an important reason for the increase. Abnormal glucose and lipid metabolism can easily damage endothelial cells and activate internal and exogenous blood coagulation pathways, leading to a significant increase in fibrinogen levels, and the increased fibrinogen makes blood sticky. Sex increases, blood flow slows down, making blood appear hypercoagulable state [5]. However, in this study, the four indexes of prothrombin were not significantly different between the two groups of patients. Only PT was related to blood lipids, which may be related to the stroke disease itself. Chen et al. [6] showed that cerebral hemorrhage can affect blood glucose, the number of white blood cells and fibrinolysis to a certain extent, while platelets and related indicators have no significant changes, indicating that elevated blood glucose in the acute phase will lead to poor prognosis. The study found that the plasma thrombin cleavage fibrinopeptide A of the patients who were studied immediately after the stroke increased significantly, the plasma concentration of the platelet release product $\beta$-hemoglobin increased slightly, and immediately increased after the stroke, and these did not occur after the infarction. Over time, it was concluded that the activity of thrombin and plasmin in plasma increased after thrombotic stroke [7]. Although there is evidence that increased fibrinogen concentration is associated with an increased risk of stroke, the role of abnormalities in the coagulation and fibrinolytic system in these processes has not been correctly assessed on clinical results. Smaller studies have found elevated FVIII/vWF associated with acute stroke and elevated tissue plasminogen activator levels. Although factor VII is considered a risk factor for coronary artery disease, little is known about its role in the development of cerebrovascular disease [8]. Gentile et al. [9] showed that the changes in blood coagulation after acute ischemic stroke in patients with diabetes and hyperglycemia, compared with non-diabetic patients, acute ischemic stroke patients with diabetes and hyperglycemia have stronger procoagulant status. Diabetic cerebral infarction does have more serious coagulation dysfunction [10]. Our study did not show that the four items of prothrombin between the two groups of patients were different, nor did it show that blood sugar had an effect on this. This may be that the acute effect of stroke is more than the chronic effect of diabetes, and the mechanism needs to be further developed in the study.

\section{Conflicts of Interest}

The authors declare no conflicts of interest regarding the publication of this paper. 


\section{References}

[1] Hu, X.L. and Wang, Z.K. (2009) Correlation Analysis on Blood Glucose, Renal Function and Blood Gas Related Markors in Severe Trauma Patients. Modern Diagnosis \& Treatment, 20, 270-272.

[2] Zheng, T., Xiao, C., He, J., et al. (2002) Study on the Coagulation Changes in Type 2 Diabetes Patients. Chinese Journal of Laboratory Medicine, 25, 290-292.

[3] Lv, Z.G. and Tang, Q. (2002) Changes of Plasma Coagulation Parameters in Aged NIDDM Patients. Journal of Jiangsu University (Medicine Edition), 12, 252-253.

[4] Lin, G.L., Huang, L.F., Qiu, S.B., et al. (2005) Coagulation Disturbance in Patients with Type 2 Diabetes Mellitus. Chinese Journal of Hemorheology, 15, 86-87, 94.

[5] Ahmed, M., Chowdhury, N.A., Sofdar, G., et al. (2014) High Fibrinogen Level Is an Independent Predictor of Presence and Severity of Coronary Artery Disease. Cardiovascular Journal, 7, 4-10. https://doi.org/10.3329/cardio.v7i1.20784

[6] Chen, Y.N., Sun, L. and Zheng, J.H. (2004) Correlation of Prognosis with the Changes of Blood Glucose and Blood Lipid and Blood Coagulation in Patients with Cerebral Hemorrhage. Chinese Journal of Clinical Rehabilitation, 8, 4512-4513.

[7] Lane, D.A., Wolff, S., Ireland, H., et al. (1983) Activation of Coagulation and Fibrinolytic Systems Following Stroke. British Journal of Haematology, 53, 655-658. https://doi.org/10.1111/j.1365-2141.1983.tb07316.x

[8] Catto, A.J. and Grant, P.J. (1995) Risk Factors for Cerebrovascular Disease and the Role of Coagulation and Fibrinolysis. Blood Coagulation \& Fibrinolysis, 6, 497-510. https://doi.org/10.1097/00001721-199509000-00001

[9] Gentile, N.T., Vaidyula, V.R., Kanamalla, U., et al. (2007) Factor VIIa and Tissue Factor Procoagulant Activity in Diabetes Mellitus after Acute Ischemic Stroke: Impact of Hyperglycemia. Thrombosis and Haemostasis, 98, 1007-1013. https://doi.org/10.1160/TH06-12-0719

[10] Zhou, J., Liu, N., Gu, Q.Y., et al. (2011) Levels and Clinical Significance of Serum Tissue Factor and Tissue Factor Pathway Inhibitor in Diabetic Stroke. Journal of Apoplexy and Nervous Diseases, 28, 420-422. 